\title{
EFICIÊNCIA DO ÓLEO DE NIM (AZADIRACHTA INDICA A. JUSS) NO CONTROLE DE BREVICORYNE BRASSICAE (LINNAEUS, 1758) E MYZUS PERSICAE (SULZER, 1776) (HEMIPTERA: APHIDIDAE) EM COUVE-MANTEIGA BRASSICA OLERACEA LINNAEUS VAR. ACEPHALA
}

\section{G.A. Carvalho, N.M. Santos, E.C. Pedroso, A.F. Torres}

Universidade Federal de Lavras, Departamento de Entomologia, CP 3037, CEP 37200-000, Lavras, MG, Brasil. E-mail- gacarval@ufla.br

\section{RESUMO}

\begin{abstract}
A planta de Nim (Azadirachta indica A. Juss) possui propriedades inseticidas e vem sendo amplamente utilizada como alternativa no controle de muitos artrópodes-praga. Sendo os pulgões Brevicoryne brassicae (Linnaeus, 1758) e Myzus persicae (Sulzer, 1776) pragas importantes para a cultura da couve-manteiga, avaliou-se neste trabalho a eficiência do extrato de óleo de Nim para o controle desses insetos. Dez pulgões adultos foram colocados em placas de Petri de $5 \mathrm{~cm}$ de diâmetro, contendo discos foliares de couve sobre uma camada de ágar-gel. Os insetos receberam as substâncias por meio de pulverizador manual, nas concentrações de $0,25 \% ; 0,5 \%$; $0,75 \% ; 1,0 \%$ e $2,0 \%$. Como testemunha positiva, utilizou-se imidaclopride $0,028 \%$ e negativa, apenas água destilada. As placas de Petri foram mantidas em câmara climatizada a $25 \pm 2^{\circ} \mathrm{C}$, umidade relativa de $70 \pm 10 \%$ e fotofase de 12 horas. As avaliações da mortalidade dos pulgões e do número de ninfas sobreviventes oriundas de adultos tratados foram realizadas a $1 \mathrm{~h}, 6 \mathrm{~h}, 12 \mathrm{~h}$, $24 \mathrm{~h}, 48 \mathrm{~h}$ e $72 \mathrm{~h}$, após a pulverização dos compostos sobre os insetos. O óleo de Nim em todas as concentrações testadas foi eficiente no controle de B. brassicae, e para M. persicae somente nas concentrações de $1 \%$ e $2 \%$.
\end{abstract}

PALAVRAS-CHAVE: Brassicaceae, inseticida botânico, afídeos, mortalidade.

\section{ABSTRACT}

EFFICIENCY OF NEEM OIL (AZADIRACHTA INDICA A. JUSS) IN THE CONTROL OF BREVICORYNE BRASSICAE (LINNAEUS, 1758) AND MYZUS PERSICAE (SULZER, 1776) (HEMIPTERA: APHIDIDAE), IN KALE (BRASSICA OLERACEA LINNAEUS VAR. ACEPHALA). The neem plant (Azadirachta indica A. Juss) possesses insecticide properties and has been used widely as an alternative in the control of a number of pest arthropods. As the aphids Brevicoryne brassicae (Linnaeus, 1758) and Myzus persicae (Sulzer, 1776) are important pests in the cultivation of kale, the present study was aimed to evaluate the efficiency of the extract of neem oil for the control of these insects. Ten adult aphids were put into Petri dishes $5 \mathrm{~cm}$ in diameter, containing leaf disk of kale on an agar gel layer. The insects were given the substances by means of a hand sprayer at the concentrations of $0.25 \% ; 0.50 \% ; 0.75 \% ; 1.0 \%$ and $2.0 \%$. As positive controls, $0.028 \%$ imidacloprid was utilized, while the negative control was only distilled water. The Petri dishes were maintained in an climatic chamber at $25 \pm 2^{\circ} \mathrm{C}$, relative humidity of $70 \pm 10 \%$ and 12-hour photophase. The evaluations of mortality of the aphids and of the number of surviving nymphs from the treated adults were carried out at $1 \mathrm{~h}, 6 \mathrm{~h}, 12 \mathrm{~h}, 24 \mathrm{~h}, 48 \mathrm{~h}$ and $72 \mathrm{~h}$ after spraying of the compounds on the insects. Neem oil at all the tested concentrations is efficient in the control of B. brassicae, but for M. persicae it is toxic only at the concentrations of $1 \%$ and $2 \%$.

KEY WORDS: Brassicaceae, botanic insecticide, aphids, mortality.

\section{INTRODUÇÃO}

Inseticidas químicos têm sido empregados na agricultura com freqüência e relativo sucesso, no entanto, podem provocar contaminação ambiental, presença de resíduos nos alimentos, efeitos prejudiciais sobre os inimigos naturais e seleção de populações de insetos resistentes. Em função disto, tem-se observado crescente interesse pelo desenvolvimento de alternativas de controle de pragas, seja através do uso 
de inimigos naturais ou de inseticidas de origem botânica.

As principais plantas das quais são obtidas substâncias com propriedades inseticidas pertencem aos gêneros Nicotiana (Solanaceae), produtoras de nicotina e nornicotina; Derris, Lonchocarpus, Tephrosia e Mundulea (Leguminoseae), produtoras de rotenóides; Chrysanthemun (Asteraceae), produtoras de piretrinas e a Azadirachta (Meliaceae), produtoras de azadiractina (LAGUNES; RodRíGUEZ, 1992).

Por apresentarem metabólitos secundários com significativa toxicidade, alguns trabalhos foram desenvolvidos visando avaliar o efeito do Nim (Azadirachta indica A. Juss) no controle de pragas, tais como: bicho-mineiro do cafeeiro Leucoptera coffeella (Guérin-Menéville, 1842) (Lepidoptera: Lyonetiidae) (MArtinez; EMdEM, 2001); mosca-das-frutas Ceratitis capitata (Wiedemann, 1824) (Diptera: Tephritidae) (NAKANO, 2001); lagarta-da-soja Anticarsia gemmatalis Hübner, 1818 (Lepidoptera: Noctuidae) e lagarta-docartucho Spodoptera frugiperda (J.E. Smith, 1797) (Lepidoptera: Noctuidae) (VIANA et al., 2000); curuquerê-do-algodoeiro Alabama argillacea (Hübner, 1818) (Lepidoptera: Noctuidae) e traça-do-tomateiro Tuta absoluta (Meyrick, 1917) (Lepidoptera: Gelechiidae) (Trindade et al., 2000; BRUnherotto; VENDRAMIM, 2001), entre outros.

A azadiractina, principal substância tóxica presente na planta de Nim, é um tetranotriterpenóide (limonóide), solúvel em água e em álcool, sensível aos raios ultravioletas, sendo eliminada do ambiente em cerca de 20 dias (Martinez, 2002). Pode causar inibição da biosíntese de quitina, deformação em pupas e adultos, redução na longevidade de adultos, alterações na atratividade dos insetos por feromônios, esterilização e inibição de oviposição e mortalidade de formas imaturas e adultas, além de afetar a reprodução dos insetos, fazendo com que eles produzam menor número de ovos ou ovos menos férteis (Koul et al., 1990; Mordue; BlacKWEll, 1993; M ordue; Nisbet, 2000; CiociolaJunior; Martinez, 2002).

Tem maior ação por ingestão, sendo responsável pela inibição alimentar de cerca de 200 espécies de insetos, com maior eficiência observada em lagartas desfolhadoras. A azadiractina pode também afetar a síntese do hormôniojuvenile, assim sendo, os insetos afetados não passam da condição farata, tornando-se imóveis e cessando a alimentação (GovindACHARI, 1992).

Em função do reduzido número de trabalhos a respeito da ação afidicida do óleo de nim, o presente estudo objetivou avaliar a sua eficiência no controle de $B$. brassicae e $M$. persicae, as principais pragas que atacam couve-manteiga B. oleracea L. var. acephala no Brasil.

\section{MATERIAL E MÉTODOS}

Os pulgões utilizados nos bioensaios foram coletados em infestações naturais em plantas de couve-manteiga $B$. oleracea var. acephala, cultivadas em campo, no Setor de Olericultura da Universidade Federal de Lavras (UFLA), em Lavras, MG.

Discos de folhas de couve com cerca de $5 \mathrm{~cm}$ de diâmetroforam colocados sobre uma camada deágargel (10 g de ágar/L de água destilada) de aproximadamente $0,5 \mathrm{~cm}$ de espessura contida em placas de Petri de $5 \mathrm{~cm}$ de diâmetro, de modo a garantir a turgidez das folhas durante o período de avaliação.

Foram colocados dez pulgões adultos por placa, com auxílio de um pincel de ponta fina sobre os discos foliares e, em seguida, os inseticidas foram aplicados com pulverizador manual (cerca de $2 \mathrm{mg}$ de calda. $\mathrm{cm}^{-2}$ ). $\mathrm{O}$ óleo de Nim (produto comercial Nim-I-Go ${ }^{\circledR}$ ) foi avaliado em diferentes concentrações $(0,25 ; 0,5 ; 0,75$; 1,0 e $2,0 \%$ ), imidaclopride $0,028 \%$ foi a testemunha positiva e água destilada a testemunha negativa.

Após as pulverizações, as placas de Petri contendo os insetos foram fechadas com filme plástico de PVC laminado, perfurado com alfinete para permitir a aeração, e mantidas em câmara climatizada à temperatura de $25 \pm 2^{\circ} \mathrm{C}$, umidade relativa de $70 \pm 10 \%$ e fotofase de 12 horas.

Utilizou-se o delineamento inteiramente casualizado, em parcelas subdivididas no tempo, com os produtos nas parcelas, sendo utilizados sete tratamentos com seis repetições, e cada parcela constituída de 10 pulgões.

As avaliações da mortalidade dos pulgões foram realizadas com 1,6,12,24,48e 72h após a pulverização, ocasião em que também foi anotado o número deninfas sobreviventes oriundas de adultos tratados. Os resultadosforam submetidos à análise de variância eas médias dos tratamentoscomparados pelotestedeScott-Knottao nível de 5\% de probabilidade (SCOTT; KNOTT, 1974).

\section{RESULTADOS E DISCUSSÃO}

\section{Toxicidade dos inseticidas para $B$. brassicae}

Verificou-se que, à medida que se aumentaram as concentrações do óleo de Nim e o tempo após a aplicação, ocorreu acréscimo de mortalidade para $B$. brassicae (Tabelas 1 e 3) (Fig. 1).

A partir de seis horas após a aplicação dos inseticidas, a concentração $2 \%$ do óleo de Nim ocasionou mortalidade semelhante à testemunha positiva (imidaclopride). Às 24h, todas as concentrações do óleo de Nim apresentaram mortalidade significativa, com média de 33,3 a 96,7\%. Após 48h das pulverizações, todas as concentrações do Nim iguais ou maio- 
res que $0,5 \%$ provocaram alta mortalidade $(88,3$ a $96,7 \%$ ) de $B$. brassicae, e às $72 \mathrm{~h}$ constatou-se que todas as concentrações causaram mortalidade acima de $85 \%$, comportando-se de forma semelhante ao imidaclopride (Tabela 1).

A ação afidicida do óleo de Nim constatada no presente trabalho também foi verificada por outros pesquisadores. VERKERK et al. (1998), ao estudarem a ação inseticida de extratos de sementes de Nim aplicados na superfície adaxial de folhas de repolho, observaram $100 \%$ de mortalidade das ninfas de $M$. persicae e B. brassicae, após $96 \mathrm{~h}$ da aplicação dos extratos. SANTOS et al. (2004) aplicaram extrato de sementes de Nim sobre pulgões $A$. gossypii, o que provocou mortalidade de ninfas, redução da longevidade e fecundidade.

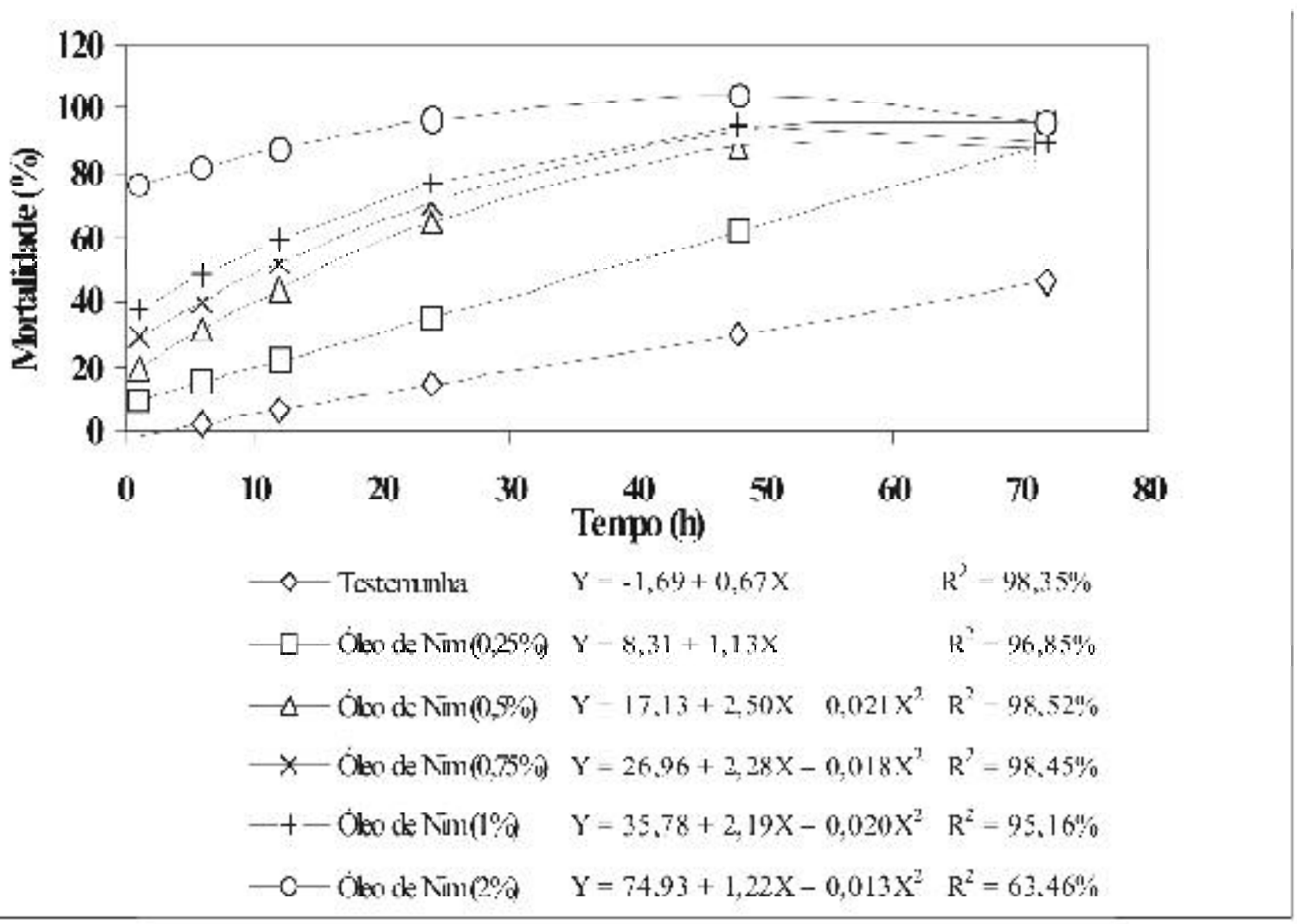

Fig. 1 - Mortalidade (\%) de B. brassicae provocada pelo óleo de Nim ao longo das avaliações. Temperatura de 25 $\pm 2^{\circ} \mathrm{C}$, UR de $70 \pm 10 \%$ e fotofase de 12 horas.

Tabela 1 - Mortalidade (\%) de B. brassicae submetidos à aplicação do óleo de Nim em diferentes concentrações. Temperatura de $25 \pm 2{ }^{\circ} \mathrm{C}$, UR de $70 \pm 10 \%$ e fotofase de 12 horas.

\begin{tabular}{lrrrrrr}
\hline Tratamento & \multicolumn{5}{c}{ Horas após a pulverização* } \\
\cline { 2 - 7 } & \multicolumn{1}{c}{1} & \multicolumn{6}{c}{6} & \multicolumn{1}{c}{12} & \multicolumn{1}{c}{24} & \multicolumn{1}{c}{48} & 72 \\
\hline Água & $1,7 \pm 0,6 \mathrm{a}$ & $1,7 \pm 0,9 \mathrm{a}$ & $6,7 \pm 1,3 \mathrm{a}$ & $10,0 \pm 2,6 \mathrm{a}$ & $31,7 \pm 5,8 \mathrm{a}$ & $46,7 \pm 3,1 \mathrm{a}$ \\
Nim 0,25\% & $5,0 \pm 1,2 \mathrm{a}$ & $11,7 \pm 2,8 \mathrm{a}$ & $30,0 \pm 5,7 \mathrm{~b}$ & $33,3 \pm 5,6 \mathrm{~b}$ & $68,3 \pm 9,4 \mathrm{~b}$ & $85,0 \pm 5,0 \mathrm{~b}$ \\
Nim 0,5\% & $15,0 \pm 3,7 \mathrm{a}$ & $35,0 \pm 6,9 \mathrm{~b}$ & $48,3 \pm 6,3 \mathrm{c}$ & $61,7 \pm 10,9 \mathrm{c}$ & $88,3 \pm 10,6 \mathrm{c}$ & $88,3 \pm 5,2 \mathrm{~b}$ \\
Nim 0,75\% & $26,7 \pm 5,3 \mathrm{~b}$ & $38,3 \pm 7,1 \mathrm{~b}$ & $58,3 \pm 7,1 \mathrm{~d}$ & $70,0 \pm 11,0 \mathrm{c}$ & $91,6 \pm 11,1 \mathrm{c}$ & $96,7 \pm 7,3 \mathrm{~b}$ \\
Nim 1\% & $33,3 \pm 6,5 \mathrm{~b}$ & $46,7 \pm 9,1 \mathrm{~b}$ & $68,3 \pm 9,8 \mathrm{~d}$ & $76,7 \pm 11,8 \mathrm{c}$ & $90,0 \pm 10,7 \mathrm{c}$ & $91,7 \pm 6,9 \mathrm{~b}$ \\
Nim 2\% & $65,0 \pm 11,8 \mathrm{c}$ & $88,3 \pm 14,6 \mathrm{c}$ & $96,7 \pm 11,5 \mathrm{e}$ & $96,7 \pm 13,1 \mathrm{~d}$ & $96,7 \pm 11,3 \mathrm{c}$ & $98,3 \pm 7,6 \mathrm{~b}$ \\
Imidaclopride & $96,7 \pm 16,4 \mathrm{~d}$ & $98,3 \pm 17,1 \mathrm{c}$ & $98,3 \pm 11,9 \mathrm{e}$ & $100,0 \pm 0,0 \mathrm{~d}$ & $100,0 \pm 0,0 \mathrm{c}$ & $100,0 \pm 0,0 \mathrm{~b}$ \\
\hline
\end{tabular}

$\mathrm{CV}(\%)=19,45$

*As médias seguidas pela mesma letra, na coluna, não diferem entre si pelo teste de Scott e Knott no nível de 5\% de probabilidade. 
Os efeitos da azadiractina sobre insetos incluem também repelência, deterrência alimentar, interrupção do crescimento, interferência na metamorfose, esterilidade e anormalidades anatômicas (MORDUE; Nisbet, 2000; M ARTINEZ; EMdEN, 2001). É provável que, no presente trabalho, o óleo de Nim também tenha causado efeito de repelência e inibição alimentar dos pulgões, provocando redução da sua longevidade pela falta de alimentação. Assim, recomenda-se que sejam feitos estudos mais detalhados para se constatar o verdadeiro efeito da azadiractina sobre essas espécies de pulgões.

A diferença no comportamento das curvas de mortalidade para cada concentração do óleo de Nim ao longo do tempo de avaliação, em relação à água, pode ser observada na Figura 1 em concentrações maiores que $0,25 \%$, demonstrando a eficiência do produto para B. brassicae.

Todas as concentrações do óleo de Nim iguais ou superiores a $0,5 \%$ apresentaram número deninfas de $B$. brassicae oriundas de adultos pulverizados semelhante ao tratamento com imidaclopride, mostrando a influência doóleo sobre a reprodução destes insetos. O óleo de Nim 0,25\% foi o único que, apesar de apresentar-se tóxico, não seigualou ao imidaclopride (Tabela 2).

Os resultados do presente trabalho confirmam os obtidos por SANTOS et al. (2004) que, ao infestarem folhas de algodoeiro tratadas com o extrato de Nim com ninfas de $A$. gossypii, constataram que as ninfas sobreviventes, quando adultas, tiveram influência negativa na sua reprodução.

\section{Toxicidade dos inseticidas para M. persicae}

A partir de 48 horas após a aplicação dos inseticidas, verificou-se mortalidade significativa de $M$. persicae nos tratamentos à base de óleo de Nim (Tabela 3).

Apesar da baixa mortalidade $(<60 \%)$ ocorrida para esta espécie, observou-se diferença no comportamento das curvas de mortalidade para os tratamentosà base deóleo deNim aolongo do tempo em relação à água, caracterizando o efeito tóxico deste produto ao M. persicae (Fig. 2). Venzon et al. (2007), ao estudarem a toxicidade letal e subletal do extrato de semente de Nim sobre adultos de M. persicae, observaram mortalidade de 55,0 e 59,1\%, nas concentrações de 0,5 e $1,0 \%$, respectivamente, sendo os resultados semelhantes ao encontrado neste trabalho. Já Hu et al. (1998) obtiveram 100\% de mortalidade de ninfas de segundo instar dos pulgões M. persicae e B. brassicae, colocando-se pecíolos de folhas decouveem recipientes contendo emulsão de Nim.

Tabela 2 - Número médio de ninfas mortas de B. brassicae oriundas de adultos tratados $72 \mathrm{~h}$, após a aplicação do óleo de Nim em diferentes concentrações. Temperatura de 25 $\pm 2^{\circ} \mathrm{C}$, UR de $70 \pm 10 \%$ e fotofase de 12 horas.

\begin{tabular}{lc}
\hline Tratamentos & B. brassicae \\
\hline Água & $12,8 \pm 1,6 \mathrm{c}$ \\
Nim $0,25 \%$ & $58,7 \pm 6,9 \mathrm{~b}$ \\
Nim $0,5 \%$ & $88,0 \pm 7,7 \mathrm{a}$ \\
Nim 0,75\% & $91,2 \pm 8,1 \mathrm{a}$ \\
Nim $1 \%$ & $94,7 \pm 8,2 \mathrm{a}$ \\
Nim 2\% & $100,0 \pm 0,0 \mathrm{a}$ \\
Imidaclopride & $100,0 \pm 0,0 \mathrm{a}$ \\
\hline & $\mathrm{CV}(\%)=38,41$ \\
\hline
\end{tabular}

*As médias seguidas pela mesma letra, na coluna, não diferem entre si pelo teste de Scott e Knott no nível de 5\% de probabilidade.

Tabela 3 - Mortalidade (\%) de M. persicae submetidos à aplicação do óleo de Nim em diferentes concentrações. Temperatura de $25 \pm 2^{\circ} \mathrm{C}$, UR de $70 \pm 10 \%$ e fotofase de 12 horas.

\begin{tabular}{lrrrrrr}
\hline Tratamento & \multicolumn{6}{c}{ Horas após a aplicação* } \\
\cline { 2 - 7 } & \multicolumn{1}{c}{1} & \multicolumn{1}{c}{6} & \multicolumn{1}{c}{12} & \multicolumn{1}{c}{48} & 72 \\
\hline Água & $0,0 \pm 0,0 \mathrm{a}$ & $1,7 \pm 0,6 \mathrm{a}$ & $6,7 \pm 1,2 \mathrm{a}$ & $8,3 \pm 2,3 \mathrm{a}$ & $10,0 \pm 2,5 \mathrm{a}$ & $10,0 \pm 1,8 \mathrm{a}$ \\
Nim $0,25 \%$ & $0,0 \pm 0,0 \mathrm{a}$ & $3,3 \pm 0,9 \mathrm{a}$ & $6,7 \pm 1,2 \mathrm{a}$ & $10,0 \pm 2,7 \mathrm{a}$ & $21,7 \pm 5,6 \mathrm{~b}$ & $31,7 \pm 2,8 \mathrm{~b}$ \\
Nim 0,5\% & $0,0 \pm 0,0 \mathrm{a}$ & $3,3 \pm 0,9 \mathrm{a}$ & $15,0 \pm 2,3 \mathrm{a}$ & $20,0 \pm 3,0 \mathrm{a}$ & $25,0 \pm 5,9 \mathrm{~b}$ & $51,7 \pm 4,4 \mathrm{c}$ \\
Nim 0,75\% & $0,0 \pm 0,0 \mathrm{a}$ & $1,7 \pm 0,5 \mathrm{a}$ & $11,7 \pm 1,9 \mathrm{a}$ & $18,3 \pm 2,9 \mathrm{a}$ & $23,3 \pm 5,7 \mathrm{~b}$ & $30,0 \pm 2,6 \mathrm{~b}$ \\
Nim 1,0\% & $0,0 \pm 0,0 \mathrm{a}$ & $5,0 \pm 1,3 \mathrm{a}$ & $16,7 \pm 2,1 \mathrm{a}$ & $23,3 \pm 3,1 \mathrm{a}$ & $30,0 \pm 6,1 \mathrm{~b}$ & $50,0 \pm 4,3 \mathrm{c}$ \\
Nim 2,0\% & $0,0 \pm 0,0 \mathrm{a}$ & $8,3 \pm 1,7 \mathrm{a}$ & $15,0 \pm 2,0 \mathrm{a}$ & $23,3 \pm 3,1 \mathrm{a}$ & $30,0 \pm 6,1 \mathrm{~b}$ & $40,0 \pm 3,6 \mathrm{c}$ \\
Imidaclopride & $83,3 \pm 8,4 \mathrm{~b}$ & $100,0 \pm 0,0 \mathrm{~b}$ & $100,0 \pm 0,0 \mathrm{~b}$ & $100,0 \pm 0,0 \mathrm{~b}$ & $100,0 \pm 0,0 \mathrm{c}$ & $100,0 \pm 0,0 \mathrm{~d}$ \\
\hline
\end{tabular}

$\mathrm{CV}(\%)=44,92$

*As médias seguidas pela mesma letra, na coluna, não diferem entre si pelo teste de Scott e Knott no nível de 5\% de probabilidade. 


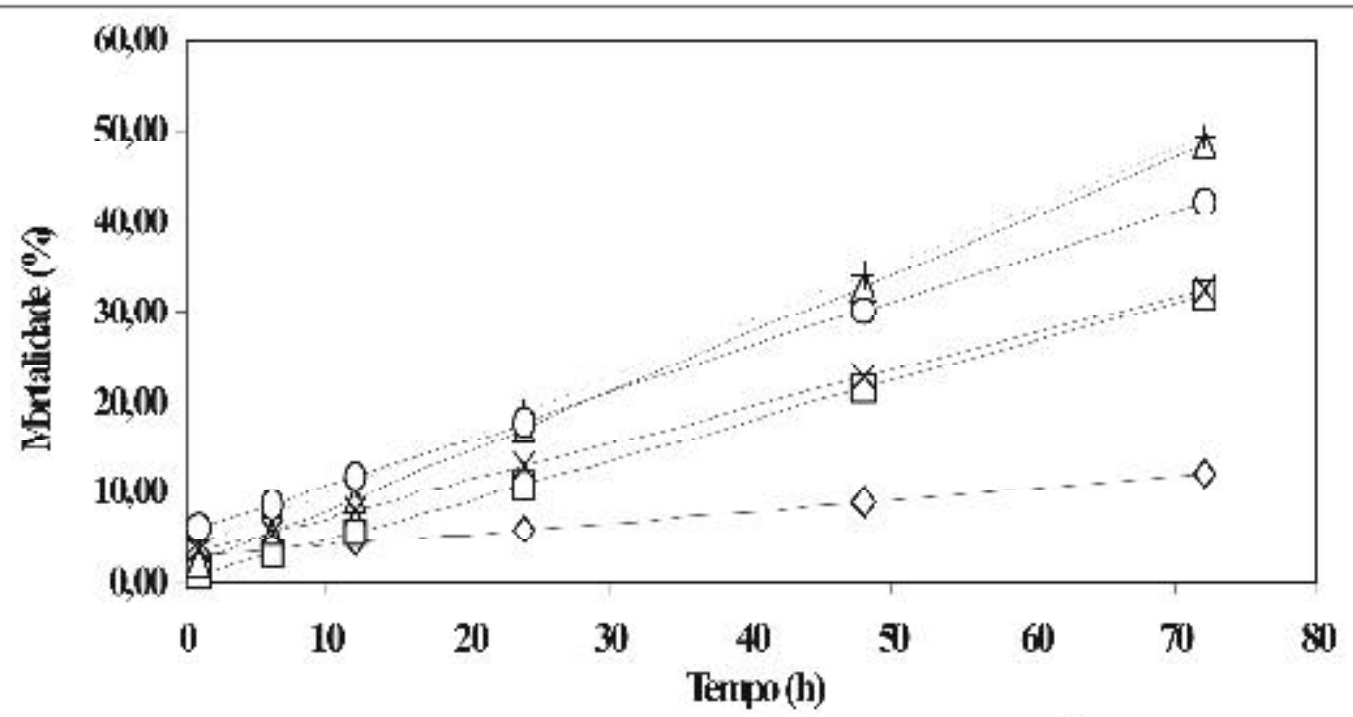

$$
\begin{aligned}
& 0 \text { lestemunha } \mathrm{Y}=2,60+0,13 \mathrm{X} \quad \mathrm{R}^{2}=69,02 \% \\
& \square-\text { OkodeNum0,29\% } \mathrm{Y}=0,35+0,44 \mathrm{X} \quad \mathrm{R}^{2}=99,63 \% \\
& \Delta \text { Obarke Ninn0, } 50 \% \quad \mathrm{Y}=1,54+0,65 \mathrm{X} \quad \mathrm{R}^{2}=93,03 \% \\
& X \text { Obercle Nim10,79\% } Y=3,06-0,41 X \quad \mathrm{~K}^{2}=89,23 \% \\
& + \text { Oxode Nmo } 1 \% \quad \mathrm{Y}=3,51+0,64 \mathrm{X} \quad \mathrm{R}^{2}=94,48 \% \\
& 0 \text { Oorale Nm2 } 2 \% \quad \mathrm{Y}=5,63+0,51 \mathrm{X} \quad \mathrm{R}^{2}=92,18 \%
\end{aligned}
$$

Fig. 2 - Mortalidade (\%) de M. persicae ao longo das avaliações. Temperatura de $25 \pm 2^{\circ} \mathrm{C}$, UR de $70 \pm 10 \%$ e fotofase de 12 horas.

Tabela 4 - Número médio de ninfas mortas deM. persicae oriundas de adultos tratados $72 \mathrm{~h}$, após a aplicação do óleo de Nim em diferentes concentrações. Temperatura de 25 $\pm 2^{\circ} \mathrm{C}$, UR de $70 \pm 10 \%$ e fotofase de 12 horas.

\begin{tabular}{lc}
\hline Tratamentos & M. persicae \\
\hline Água & $57,8 \pm 1,7 \mathrm{c}$ \\
Nim $0,25 \%$ & $64,8 \pm 2,0 \mathrm{c}$ \\
Nim $0,5 \%$ & $71,6 \pm 2,5 \mathrm{c}$ \\
Nim 0,75\% & $70,5 \pm 2,3 \mathrm{c}$ \\
Nim 1\% & $86,0 \pm 3,7 \mathrm{~b}$ \\
Nim 2\% & $79,1 \pm 3,4 \mathrm{~b}$ \\
Imidaclopride & $100,0 \pm 0,0 \mathrm{a}$ \\
\hline & $\mathrm{CV}(\%)=40,52$
\end{tabular}

*As médias seguidas pela mesma letra, na coluna, não diferem entre si pelo teste de Scott e Knott no nível de 5\% de probabilidade.

Em relação ao número de ninfas provenientes de adultos tratados deM.persicae, verificou-se que ó óleo de Nim apresentou eficiência somente em concentrações superiores a 1,0\% (Tabela 4). VENZON et al. (2007) observaram que as ninfas de M. persicae, oriundas de adultos tratados com extratos de semente de Nim a 0,5e1,0\%, apresentaram mortalidade de 79,4 e $82,6 \%$, respectivamente.

De modo geral, a espécie $B$. brassicae foi mais susceptível aos efeitos do óleo de Nim que $M$. persicae.

Novos testes em condições de semicampo e campo devem ser realizados para verificar a eficiência agronômica do produto no controle desses pulgões.

\section{CONCLUSÕES}

Oóleo de Nim em todas as concentrações testadas é eficiente no controle de B. brassicae.

O óleo de Nim é tóxico para $M$. persicae somente em concentrações iguais ou maiores que 1\%, não ultrapassando $60 \%$ de mortalidade.

A espécie B.brassicae émais susceptível aos efeitos do óleo de Nim do que $M$. persicae.

Imidaclopride é tóxico para os pulgões M.persicae e B. brassicae. 


\section{AGRADECIMENTOS}

À Fundação de Amparo à Pesquisa do Estado de Minas Gerais (FAPEMIG).

\section{REFERÊNCIAS}

BRUNHEROTTO, R.; VENDRAMIM, J.D. Bioatividade de extratos aquosos de Melia azedarach L. e Azadirachta indica A. Juss. (Meliaceae) sobre o desenvolvimento de Tuta absoluta (Meyrick) (Lepidoptera: Gelechiidae) em tomateiro. Neotropical Entomology, v.30, n.3, p.455-459, 2001.

CIOCIOLA JUNIOR, A.I.; MARTINEZ, S.S. Nim: alternativa no controle de pragas e doenças. Belo Horizonte: EPAMIG, 2002. 24p. (Boletim Técnico n.67).

GOVINDACHARI, T.R. Chemical and biological investigations on Azadirachta indica (the neem tree). Current Science, v.63, n.3, p.117-122, 1992.

HU, M.; KLOCKE, J.A.; BARNBY, M.A.; CHIU, S.; ZHAO, S.; HU, M.Y. Systemic insecticidal action of azadirachtin, neem seed and chinaberry seed extracts applied as soil drenches to potted plants. Entomologia Sinica, v.5, n.2, p.177-188, 1998.

KOUL, O.; ISMAN, M.B.; KETKAR, C.M. Properties and uses of neem, Azadirachta indica. Canadian Journal of Botany, v.68, n.1, p.1-11, 1990.

LAGUNES, T.A.; RODRÍGUEZ, H. Los extractos acuosos vegetables con actividad insecticida: el combate de la conchuela del frijol. Texcoco: USAID-CONACYT-SME-CP, 1992. 57p. (Temas Selectos de Manejo de Insecticidas Agrícolas, 3).

MARTINEZ, S.S. (Ed.). O nim - Azadirachta indica: natureza, usos múltiplos, produção. Londrina: IAPAR, 2002. 142p.

MARTINEZ, S.S.; VAN EMDEM, H.F. Growth disruption, abnormalities and mortality of Spodoptera littoralis (Boisduval) (Lepidoptera: Noctuidae) caused by Azadirachtin. Neotropical Entomology, v.30, n.1, p.113$125,2001$.

MORDUE, A.J.; BLACKWELL, A. Azadirachtin: an update. Journal of Insect Physiology, v.39, n.11, p.903-924, 1993.
MORDUE, A.J.; NISBET, A. Azadirachtin from the Neem tree Azadirachta indica: its actins against insects. Anais da Sociedade Entomológica do Brasil, v.29, n.4, p.615632, 2000.

NAKANO, O. Ensaio visando o controle da mosca das frutas Ceratitis capitata com inseticidas na forma de isca visando o efeito esterilizante. In: CURSO DE CULTIVO E UTILIZAÇÃO DO NIM, 4., 2001, Piracicaba. Anais. Piracicaba: ESALQ, 2001.

SANTOS, T.M.; COSTA, N.P.; BOIÇA JÚNIOR, A.L. Efeito de extrato de nim sobre o pulgão-do-algodoeiro. Pesquisa Agropecuária Brasileira, v.39, p.1071-1076, 2004.

SCOTT, A.J.; KNOTT, M.A. A cluster analysis method for grouping means in the analysis of variance. Biometrics, v.30, n.3, p.507-512, 1974.

TRINDADE, F.C.P.; MARQUES, I.M.R.; XAVIER, H.S.; OLIVEIRA, J.V. de Extrato metanólico da amêndoa da semente de nim e a mortalidade de ovos e lagartas da traça-do-tomateiro. Scientia Agrícola, v.57, n.3, p.407413, 2000.

VENZON, M.; ROSADO, M.C.; PALLINI, A.; FIALHO, A.; PEREIRA, C.J. Toxicidade letal e subletal do nim sobre o pulgão-verde e seu predador Eriopis connexa. Pesquisa Agropecuária Brasileira, v.42, n.5, p.627-631, 2007.

VERKERK, R.H.J.; NEUGEBAUER, K.R.; ELLIS, P.R.; WRIGHT, D.J. Aphids on cabbage: tritrophic and selective insecticide interactions. Bulletin Entomological Research, v.88, p.343-349, 1998.

VIANA, P.A.; PRATES, H.T.; CRUZ, I.; WAQUIL, J.M. The effect of aqueous extract of Azadirachta indica leaves on the control of Spodoptera frugiperda fed with corn leaves. In: INTERNATIONAL CONGRESS OF ENTOMOLOGY, 21.; BRAZILIAN CONGRESS OF ENTOMOLOGY, 18., 2000, Foz do Iguaçu. Abstracts. Londrina: Embrapa Soja, 2000. v.1, p.356.

Recebido em 11/5/07

Aceito em 16/5/08 